\title{
Implementation of new Cryptographic Encryption Approach for Trust as a Service (TAAS) in Cloud Environment
}

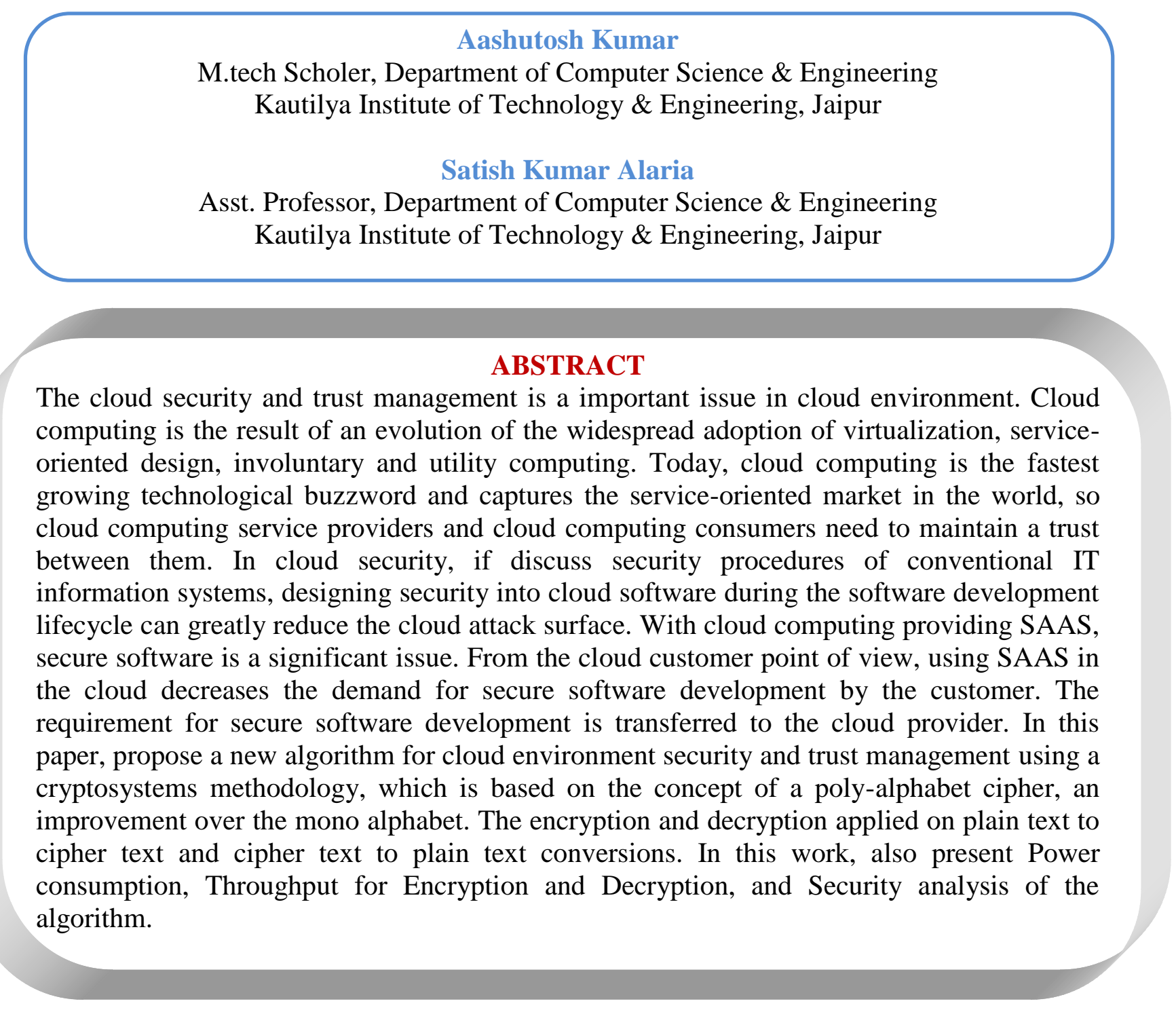

\section{INTRODUCTION}

The Internet is spread throughout the world. The Internet is used for different purposes in different domains. The actual meaning of using the Internet is changing these days. The way people compute and even the types of computers they use are more advanced than their predecessors. In the previous days of Internet, services were very efficient, unlike the current situation. Internet services are continuously improving these days and the aim is to give services according to the user demand. But systems with limited storage and computing capacity for running these services are not sufficiently capable. If a user installs any heavy software or wants better computing performance, then he/she often suffer due to the inefficient hardware support [1]. 
DOI: https://dx.doi.org/10.26808/rs.ca.i8v4.03 International Journal of Computer Application (2250-1797)

Issue 8 Volume 4, July-August 2018

TABLE 1.1: Cloud Computing Timeline

\begin{tabular}{|l|l|l|}
\hline Decade & Characteristics & Notes \\
\hline 1960 s & $\begin{array}{l}\text { McCarthyes concept \& other academic } \\
\text { research }\end{array}$ & \\
\hline $1970 s$ & Mainframe timesharing, ARPANET & IBM \& the "Seven dwarfs"- \\
\hline $1980 s$ & $\begin{array}{l}\text { ARPANET and the emergence of thin } \\
\text { client }\end{array}$ & $\begin{array}{l}\text { NeXT, Object Oriented Programming } \\
\text { and GUIs }\end{array}$ \\
\hline 1990 s & Hotmail, Salesforce, Peer1 Hosting & SaaS emerging, Net Centric trademark \\
\hline $2000 s$ & $\begin{array}{l}\text { Amazon Web Service, Rackspace, } \\
\text { DropBox \& others emerge }\end{array}$ & Cloud Revenue estimated at US\$ 58.6 \\
\hline $2010 s$ & $\begin{array}{l}\text { Google Drive, Amazon Cloud Drive, LG } \\
\text { Cloud, icloud, Smartphones }\end{array}$ & $\begin{array}{l}1 \text { new Server added to the cloud for } \\
\text { every 600 Smartphones. }\end{array}$ \\
\hline
\end{tabular}

Cloud Computing has solved all these problems. Cloud computing enables the end-user and provide better computing experience for a user. Cloud computing is an upgrade over technologies like parallel computing, distributed computing, or grid-computing.

\subsection{General Overview}

The term Cloud indicates the combination of a computer system and server to make the services accessible with the help of the Internet. This collection of server may be controlled and managed by a third-party service provider, which may be located anywhere around the world. Cloud computing service providers deliver many hosted services that are often requested by the client [2]. The National Institute of Standards and Technology (NIST) define Cloud computing, it's services and deployment models of the cloud computing paradigm. According to the visual model of NIST's working definition of Cloud Computing:

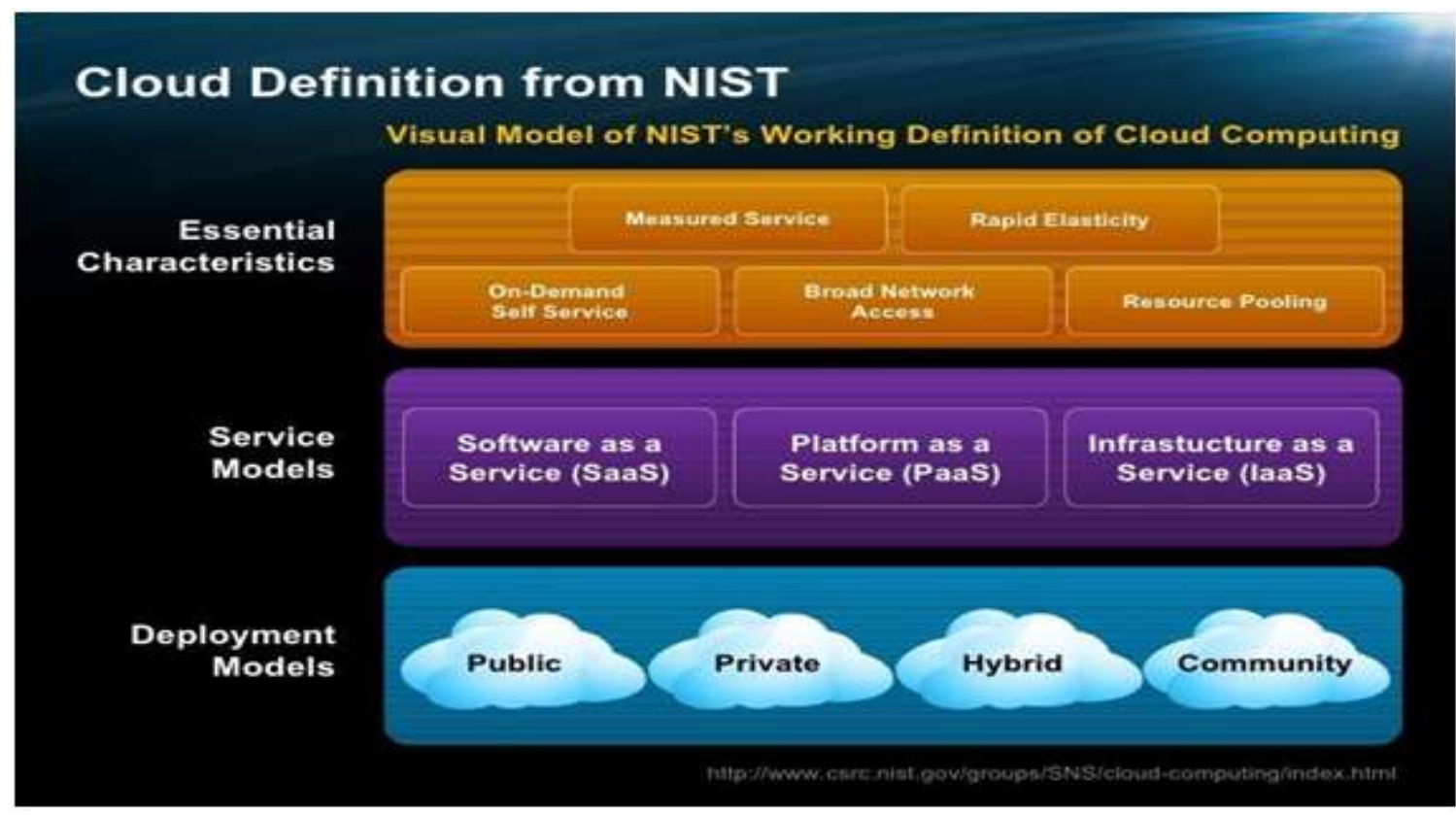

Figure 1.1 NIST Visual Model of Cloud Computing Definition 
DOI: https://dx.doi.org/10.26808/rs.ca.i8v4.03 International Journal of Computer Application (2250-1797)

Issue 8 Volume 4, July-August 2018

"Cloud computing is a paradigm that provides, on-demand network access to a shared pool of configurable computing resources (e.g., networks, servers, services, storage and applications) that can be rapidly provisioned and delivered with least management effort or service provider interaction" [2]. The cloud computing model offers cost saving benefits combined with increased use of IT. It is recommended that government \& industry should start using this technology to counter difficult economic conditions. Cloud computing is currently used but its security, interoperability are major hindrances for its extensive use.

\subsection{Essential Characteristics of Cloud Computing}

There are many characteristics for Cloud Computing, which are explained below:

\subsubsection{On-Demand Self-Service}

A client will unilaterally provision computing capabilities, like server time and network storage, as required automatically without requiring human interaction with every service supplier [2].

\subsubsection{Broad Network Access}

Capabilities are available over the network and accessed through commonplace mechanisms that promote use by heterogeneous thin or thick client platforms (e.g., mobile phones, tablets, laptops, and workstations) [2].

\subsubsection{Resource Pooling}

The provider's computing resources are pooled to serve multiple customers using a multitenant model, with various virtual and physical resources dynamically assigned and reassigned according to consumer demand. There is a sense of location-independence, the customer usually has no knowledge or control over the exact location of the provided resources but may be suitable to specify location at a higher level of abstraction (e.g., country or state). Examples of resources comprise storage memory and network bandwidth [2].

\subsubsection{Rapid Elasticity}

Abilities can be elastically provisioned and released, in some cases automatically, to scale outward and inward, proportional with demand. To the customer, the abilities available for provisioning often appear to be unlimited \& can be appropriated in any amount at any time [2].

\subsubsection{Measured Service}

Cloud systems automatically manage and optimize resource use by leveraging a metering capability at some level of abstraction relevant to the type of service (e.g. bandwidth, active user accounts, storage, and processing). Resource usage can be controlled, monitored, and reported providing transparency to both the provider and consumer of the utilized service [2].

\subsection{Problem Statement}

The prime focus of any organization is security and it is the key to the Cloud success. As many surveys show, security in the cloud and Trust management is now the main challenge of cloud computing. Trust is one of the most important part for the adoption and growth of cloud computing. Since cloud computing is widely used a technique, so the new problem arises according to its demand. The grave concern is on security including service availability; data confidentiality and provider lock-in. The fundamental ideas of cloud computing are not fresh. Cloud computing is not an advancement of technology but a new 
mode of its operation. Therefore cloud computing is compared to many existing technologies. A new problem arises due to the essence of cloud computing like security etc. In the previous algorithm, statistics or system is controlled by third-party. Third party created by cloud service provider. When user store data in the cloud, always remains in a cloud. User cannot be assured that after user delete data, it will not exist anymore, anywhere. It observes less security for stored data. In this work, propose a new encryption- decryption algorithm over previous algorithm for throughput and power consumption and trust as a service. The algorithms which are already in use can't fulfill the expectations of users. In previous algorithms, there are many problems, i.e., small key size, the high execution time for encryption and decryption at different data sizes, low throughput, and high power consumption. These algorithms have some of the issues that are resolved in proposed algorithm. In proposed work an algorithm is developed. The first algorithm uses a matrix key which on multiplication with a quaternary vector and applying a sign function on the product generates a sequence. This sequence will be used to create three distinct models of substitution method. Then the algorithm is considered to be a substitution algorithm which uses a single key to be shared by both the sender and receiver, and the cipher processes the input element continuously, generating output one element at a time. The new encryption algorithm is based on the concept of Poly alphabet cipher which is an improvement over the mono alphabet.

\section{LITERATURE SURVEY}

The Security is a principal concern when entrusting organizations critical information to geographically dispersed cloud platforms, not under the direct control of that organization. If we discuss our conventional IT information system security procedures, designing security into cloud software during the software development lifecycle can greatly reduce the cloud attack surface. From the cloud consumer's point of view, using SAAS in the cloud reduces the need for secure software development by the customer. The requirement for secure software development is transferred to the cloud provider.

Cloud computing is the next generation technology that gives us a facility to on-demand, reliable, secure in software, applications, and infrastructure as a service. The cloud computing is also known as Fifth utility service like water, electricity, energy [4].

- Trust plays a role only when the environment is uncertain and risky.

- Trust is the basis based on which certain decisions are made.

- Trust is formed using earlier knowledge, practice, and experience.

- Trust is a subjective concept depends on evaluation and values of an individual.

- Trust depends on time and new knowledge while experience will have overriding importance over the old ones.

- Trust is multi-faceted.

- Trust is context-dependent.

G. Ramesh and R. Umarani et al. [31] the hacking is the greatest problem in the wireless local area network (WLAN). Many algorithms like RC6, UMARAM, DES, 3DES, RC2 and UR5 have been used to prevent the outside attacks to eavesdrop or prevent the data to be transferred to the end-user correctly. In this paper, we study the six most common and popular symmetric cryptographic algorithms like RC6, UMARAM, DES, 3DES, RC2 and UR5. This paper analyzes their security issues and then compares their efficiency for encrypting text and image across different widely used Operating Systems. The simulation results concluded the performance of most common encryption across the different platforms. 
DOI: https://dx.doi.org/10.26808/rs.ca.i8v4.03 International Journal of Computer Application (2250-1797)

Issue 8 Volume 4, July-August 2018

Which algorithm performs better on which operating system for encrypting what kind of data.

Saket Maskara1, Mudit Saraf and Priya G et al. [32] Cloud computing has pulled in light of a legitimate concern for the numerous specialists, each - inside the instructive world and in this manner, the business - in light of the fact that it is giving numerous chances to associations by giving changed figuring administrations. For cloud computing to wind up wide embraced by each the undertakings and individuals, numerous issues must be constrained to be settled. A key issue that needs exceptional consideration is security of trust, and trust administration is a key some portion of cloud security. This paper, the creators cross-check what trust is and the way trust has been connected in appropriated registering. Trust models anticipated for fluctuated circulated framework has then been condensed. The trust administration frameworks anticipated for cloud computing are explored with unique weight on their ability, congruity in sensible heterogeneous cloud setting and implementabilty.

R.Gowthami Saranya and A.Kousalya et al. [33] Cloud computing is a word which is describe different computing concepts which contains hugenumber of computers attached through a real-time communication like internet. Cloud computing is also called distributed computing over the network i.e. the ability to execute an application or a program on many computers at thesame time.Cloud Computing is an emerging technology in today's business era. It allows convenient on demand access to resources that involve large number of computers connected through Internet. Public clouds vendors offer many resources such as application, storage, hardware, software's etc. The security issues present in public cloud is more challenging. As everything is accessed publically; many users have the threat to store and retrieve it publically. As many organizations are moving data to the cloud there is a need to protect data against unauthorized access. Hence it is necessary to study the security issues in public cloud to secure data. The purpose of this paper is to provide an overview of pubic cloud computing and the security issues involved.This paper deals with the different algorithms or method used for securing data in public cloud.

\section{METHODOLOGY}

The new generation is full of challenges and everybody wants to optimize his work by using various technologies. The amount of data to be stored may be very large or just for some time or for a definite purpose we need some software for accessing our data. To manage these problems and cloud computing gives an ultimate solution. Generally, security requires in every organization and institutes. Nowadays, internet spread like fire in the forest. Every next person is using it for various purposes. These internet based applications are beneficial to us because they are taking less time and give wonderful results. Now we will discuss previous algorithms in details.

\subsection{Data Encryption Standard (DES)}

The Data Encryption Standard (DES) is a symmetric-key algorithm for the encryption data. Although now considered insecure, it was highly influential in the advancement of modern cryptography. DES is now studied is not secure for many applications. This is essentially due to the 56-bit key size being too small; in January 1999, distributed.net and the Electronic Frontier Foundation collaborated to publicly crack a DES key in 22 hours and 15 minute [20].DES is a block cipher. DES encrypts the data in a block of 64 bits, which produces 64bit cipher text. The key length is 56 bits. Initially, the key is consisting of 64 bits. The bit position $8,16,24,32,40,48,56,64$ discarded from the key length. The Same key used both 
encryption and decryption. DES consists of 16 steps, each of which called as a Round [21]. In 1998 a special-purpose machine for "cracking DES" was designed by the Electronic Frontier Foundation for under $\$ 250,000$. In one indication, it obtained the key to an encrypted message in less than a day in conjunction with a coalition of computer users around the world [22-23].

\subsection{TRIPLE DES}

Two main variations in DES have emerged, which are double DES and triple DES. Although the meet-in-middle attack on double DES is not quite practical yet, in cryptography, it is always better to take the minimum possible changes. Consequently double DES seemed inadequate, then arise new algorithm that is called triple DES [21]. Triple DES is DES -three times. It comes in two flavors: One that uses three keys and other that uses two keys. The Idea of 3-DES is shown in the fig. The plaintext block $\mathrm{P}$ is first encrypted with a key $\mathrm{K} 1$, then encrypted with second key $\mathrm{K} 2$, and ultimately with third key $\mathrm{K} 3$, where $\mathrm{K} 1$, $\mathrm{K} 2$, and $\mathrm{K} 3$ are distinct from each other. To decrypt the cipher text $\mathrm{C}$ and receive the plain text, we require executing the operation $\mathrm{P}=\mathrm{DK} 3$ (DK2 (DK1(C))). But in Triple DES two keys is applied in algorithm [23].

TABLE 3.1: Comparison between different algorithms [30]

\begin{tabular}{|l|l|l|l|}
\hline S. No. & FACTORS & DES & 3DES \\
\hline $\mathbf{1}$ & KEY LENGTH & 56 BITS & 168 BITS \\
\hline $\mathbf{2}$ & CIPHER TYPE & $\begin{array}{l}\text { SYMMETRIC } \\
\text { ALGORITHM }\end{array}$ & $\begin{array}{l}\text { SYMMETRIC } \\
\text { ALGORITHM }\end{array}$ \\
\hline $\mathbf{3}$ & BLOCK SIZE & 64 BITS & 64 BITS \\
\hline $\mathbf{4}$ & DEVELOPED & 1977 & 1978 \\
\hline $\mathbf{5}$ & KEY & SINGLE & $\begin{array}{l}\text { SINGLE (Later divided in } 3 \\
\text { parts) }\end{array}$ \\
\hline $\mathbf{6}$ & POSSIBLE KEYS & 256 & $2112,2^{168}$ \\
\hline $\mathbf{7}$ & THROUGHPUT (MB/sec) & $3.7074 / 4.0802$ & $2.3463 / 3.5978$ \\
\hline $\mathbf{8}$ & SECryption/Decryption) & & 48 \\
\hline $\mathbf{9}$ & ROUND & PROVEN INADEQUATE & WEAK \\
\hline
\end{tabular}

\section{PROBLEM DFINITION \& PROPOSED WORK}

In proposed work, the client or user interacts with the third party auditor. The third party auditor is an authorized person appointed by the owner of the cloud (Cloud Service Provider). Then for trust as a service proposed new research for cloud computing security and research work towards designing a new cryptographic algorithm follows the sequence of steps.

1. Definition of the problem statement.

2. An algorithm 1 which involves multiplication of Quaternary vector matrix of size $256 \mathrm{x}$ 4 and a random matrix of size $4 \times 4$ then processing the output of this multiplication operation to produce the secret key. The process involved in the development of this algorithm is aimed to obtain an optimal key. 
DOI: https://dx.doi.org/10.26808/rs.ca.i8v4.03

3. An algorithm 2 which is aimed at encryption from processing plain text to produce the cipher text with the secret key generated in Algorithm 1. The cipher text will follow the transmission key holding the useful data of secret key for decryption at receiver end and will be sent along with the Cipher Text at the Transmitteres end.

4. Training of the Developed algorithms with distinct keys.

5. Adopting a proper method to recognize any garbled key while sending from the Key distribution center.

6. Comparative analysis of the developed algorithms in terms of Throughput for encryption and decryption \& Power consumption \& strength \& Security analysis.

7. Summary \& Conclusion of the work.

\subsection{Problem Definition}

All encryption algorithms rely on 2 common principles. The first is Substitution, during which every part within the plaintext is mapped to a different part. The second is Transposition during which the elements within the plaintext are rearranged. Most systems need multiple stages of substitution and transpositions. Technologist introduced confusion and diffusion. Confusion could be a technique of making certain that a cipher text offers no clue concerning the plain text and Diffusion will increase redundancy of plain text by spreading it across rows and columns.

If the sender and receiver use a similar key, the system is named as symmetric, single key, secret key or typical encryption. If the sender and therefore the receiver every uses a distinct key, the system is named as asymmetric, 2 key or public key encryption.

A block cipher creates the input one block of parts at a time, generating an output block for every input block. A stream cipher processes the input part continuously, producing output one part at a time, because it goes on.

\subsection{Proposed Algorithm}

The first algorithm uses a matrix key which on multiplication with a quaternary vector and using a sign function on the result produces a sequence. This sequence will be utilized to create 3 distinct models of substitution method. Thus the algorithm is examined to be a substitution algorithm which utilizes a single key to be shared by both the sender and receiver, and the cipher create the input element regularly, generating output one element at a time. The Developed algorithm depends on the idea of Poly alphabet cipher which is an improvement over the mono alphabet.

\subsubsection{Algorithm for producing the sequence}

$\mathrm{STEP \# 1.} \mathrm{Let} \mathrm{the} \mathrm{sequence} \mathrm{for} 0$ to $\mathrm{N}$ values where $\mathrm{N}$ is a positive integer.

STEP\#2. Transform each element of the sequence into Quaternary form of a given digit number.

STEP\#3. Represent the values of STEP\#2 in a matrix form of 256X4. STEP\#4. Subtract 1 from each element of the matrix defined in STEP\#3. STEP\#5. Consider a random matrix key of size (4X4).

STEP\#6. The output of STEP\#4 multiply with the output of STEP\#5.

STEP\#7. Convert all positive values of the matrix $(256 \times 4)$ to 1 , negative values to -1 and zero by 0 .

STEP\#8. Add 1 to each element of the output of STEP\#7.

STEP\#9. Convert Quaternary values of STEP\#8 into decimal form. A sequence is generated. 


\subsubsection{Features of New Algorithm}

1. A set of polyalphabetic substitution rule is used.

2. A new block cipher is developed.

3. A Random matrix is being used for the secret key.

4. A new coding methodology.

\subsubsection{Advantages of New Algorithm}

1. Even if the algorithm is known, it is computationally infeasible to generate matrix key.

2. Versatile to users: Different users of the internet can use different modified versions of the new algorithm. Since in this algorithm, the sign function is used, it is supposed to be strong enough.

3. As per the matrix, the same character is substituted by different alphanumerical values which provide more security for the message.

\subsubsection{Complexity of The New Algorithm}

Complexity can also be represented as orders of magnitude. If the length of the key is $\mathrm{k}$, then the complexity is presented as $2 \mathrm{k}$. It indicates that $2 \mathrm{k}$ operations are needed to break the algorithm. In the presented algorithm, a matrix key is used. This matrix key is multiplied with a quaternary vector. On the generated values a sign function is applied to change all positive values to 1 , negative values to -1 and zero to 0 . This presents the essential strength of the algorithm. Thus recognized the algorithm, known the cipher text it is quite difficult to create the matrix key. In the present algorithm, there are no means by which the key can be identified, other than trying all the combinations of key, the complexity of the algorithm is said to be exponential in quality.

\section{IMPLEMENTATION \& RESULT ANALYSIS}

Cloud computing is a key technology of the present world. A lot of research is going on in this technology. In spite of this research, there are some aspects in the wake of the fact that most of the organizations and the clients of the cloud are yet not so confident of moving into this new technical paradigm that is known as the cloud. Therefore by security point of view all the existing systems is not that much reliable. We have compared our proposed algorithm with other existing algorithms on the basis of some parameters. In order to justify that our proposed work is more efficient than others, we have introduced new encryption technique which reduces execution time for encryption and decryption. It also provides high throughput and low power consumption with increased security.

\subsection{Implementation}

Physically implementing our research work, the output shows the reduction in time and increasing security. Below are the screenshots attached for justification.

1. First of all, enter plain text as input from user.

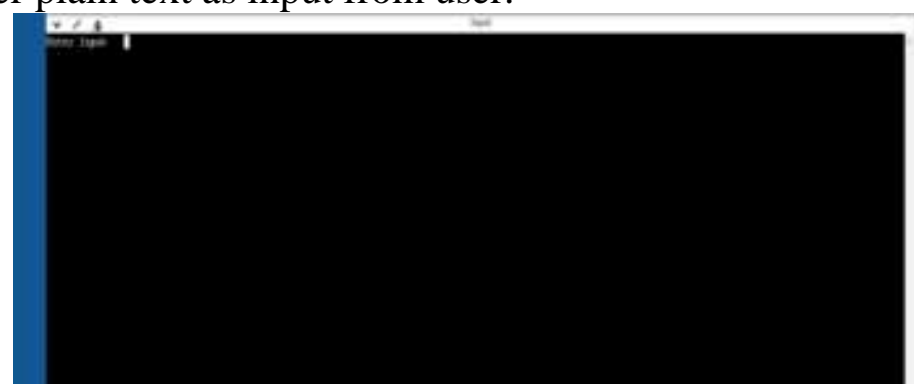

Figure 5.1 Screenshot for Entering Plain Text 
DOI: https://dx.doi.org/10.26808/rs.ca.i8v4.03 International Journal of Computer Application (2250-1797)

Issue 8 Volume 4, July-August 2018

2. Then from the input calculate word count and according to value of word count perform MOD operation, then perform some additional steps on input data and generate cipher text, each element of cipher text is separated by ? (Question mark) and . (dot) according to word count value.

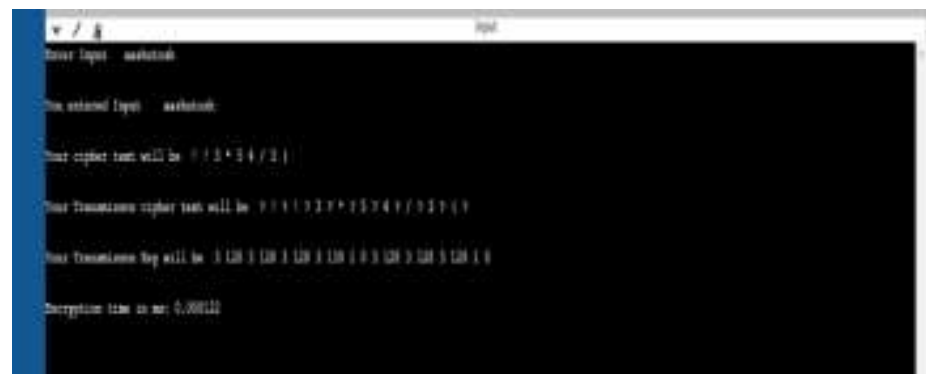

Figure 5.2 Screenshot for Generating Cipher Text

3. After generating cipher text, according to word count apply MOD inverse operation and some additional steps to retrieve plain text.

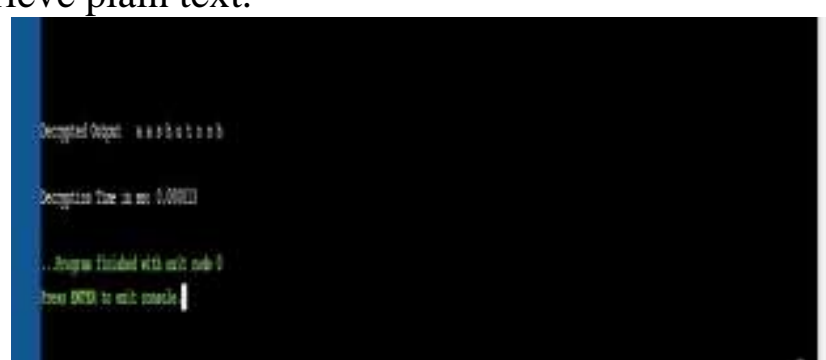

Figure 5.3 Screenshot for Retrieving Plain Text

4. This working can also be explained using Data flow diagram in Fig. 5.4

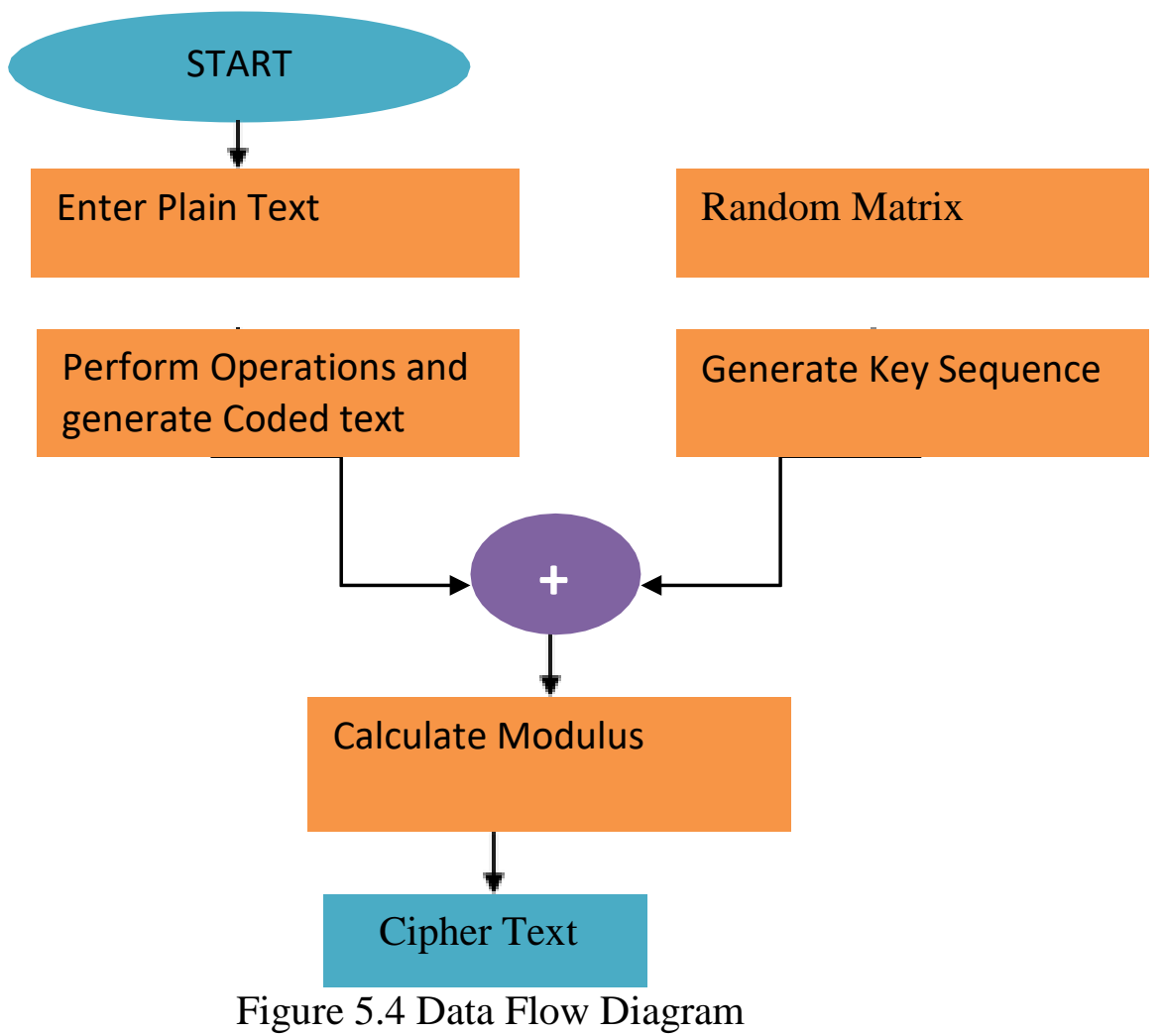


- In figure 5.4, first enter Plain Text then perform operations on Plain text and generate coded text.

- Along with this Generate Random Matrix and create key sequence.

- Calculate modulus of result from above steps \& finally generate Cipher Text.

\subsection{Comparative Analysis}

In this part [30] we have compared our work with other existing techniques. We have represented our result in the context of different parameters like:

1. Calculating execution time for different input size for encryption and decryption.

2. Calculating Throughput and Power Consumption for various algorithms.

3. Security Analysis.

In Table 5.1, we have calculated execution time (in msec) for different input data size like 49 kbytes, 59 kbytes, 100 kbytes, 247 kbytes, 321 kbytes, 694 kbytes and 899 kbytes.

After analyzing this output 10 times for each input size of data, we calculated average time for each input size.

Then we calculated average of time for all input size data, which is our execution time in (msec) for encryption.

TABLE 5.1 : Calculate Execution Time (msec) For Encryption

\begin{tabular}{|c|c|c|c|c|c|c|c|}
\hline \multirow{2}{*}{ S. No. } & \multicolumn{7}{|c|}{ Execution Time (msec) For Encryption } \\
\hline & $\begin{array}{c}49 \\
\text { kbytes }\end{array}$ & $\begin{array}{c}59 \\
\text { Kbytes }\end{array}$ & $\begin{array}{c}100 \\
\text { Kbytes }\end{array}$ & $\begin{array}{c}247 \\
\text { kbytes }\end{array}$ & $\begin{array}{c}321 \\
\text { kbytes }\end{array}$ & $\begin{array}{c}694 \\
\text { kbytes }\end{array}$ & $\begin{array}{c}899 \\
\text { Kbytes }\end{array}$ \\
\hline $\mathbf{1}$ & 10 & 10 & 20 & 60 & 80 & 170 & 260 \\
\hline 2 & 10 & 10 & 20 & 60 & 80 & 160 & 220 \\
\hline 3 & 10 & 10 & 20 & 50 & 70 & 180 & 290 \\
\hline 4 & 10 & 10 & 20 & 50 & 80 & 170 & 280 \\
\hline 5 & 10 & 10 & 20 & 60 & 70 & 160 & 230 \\
\hline 6 & 10 & 10 & 20 & 50 & 70 & 170 & 250 \\
\hline 7 & 10 & 10 & 20 & 60 & 70 & 180 & 260 \\
\hline 8 & 10 & 10 & 20 & 60 & 70 & 170 & 250 \\
\hline 9 & 10 & 10 & 20 & 60 & 70 & 160 & 250 \\
\hline 10 & 10 & 10 & 20 & 60 & 80 & 170 & 220 \\
\hline Average & 10 & 10 & 20 & 57 & 74 & 169 & 251 \\
\hline \begin{tabular}{|l} 
Average \\
Total \\
Time \\
(msec)
\end{tabular} & $84.42(\mathrm{~ms}$ & & & & & & \\
\hline
\end{tabular}


In Table 5.2, we have calculated execution time (in $\mathrm{msec}$ ) for different input data size like 49 kbytes, 59 kbytes, 100 kbytes, 247 kbytes, 321 kbytes, 694 kbytes and 899 kbytes.

After analyzing this output 10 times for each input size of data, we calculated average time for each input size.

Then we calculated average of time for all input size data, which is our execution time in (msec) for decryption.

TABLE 5.2: Calculate Execution Time (msec) For Decryption

\begin{tabular}{|c|c|c|c|c|c|c|c|}
\hline \multirow{2}{*}{ S.No. } & \multicolumn{7}{|c|}{ Execution Time (msec) For Decryption } \\
\hline & $\begin{array}{c}49 \\
\text { kbytes }\end{array}$ & $\begin{array}{c}59 \\
\text { kbytes }\end{array}$ & $\begin{array}{c}100 \\
\text { kbytes }\end{array}$ & $\begin{array}{c}247 \\
\text { kbytes }\end{array}$ & $\begin{array}{c}321 \\
\text { kbytes }\end{array}$ & $\begin{array}{c}694 \\
\text { kbytes }\end{array}$ & $\begin{array}{c}899 \\
\text { Kbytes }\end{array}$ \\
\hline 1 & 10 & 10 & 20 & 60 & 90 & 90 & 200 \\
\hline 2 & 10 & 10 & 20 & 50 & 80 & 160 & 100 \\
\hline 3 & 10 & 10 & 20 & 70 & 80 & 150 & 170 \\
\hline 4 & 10 & 10 & 20 & 80 & 70 & 180 & 180 \\
\hline 5 & 10 & 10 & 20 & 60 & 90 & 160 & 230 \\
\hline 6 & 10 & 10 & 20 & 80 & 90 & 100 & 210 \\
\hline 7 & 10 & 10 & 20 & 70 & 70 & 160 & 210 \\
\hline 8 & 10 & 10 & 20 & 70 & 70 & 150 & 220 \\
\hline 9 & 10 & 10 & 20 & 60 & 70 & 150 & 210 \\
\hline 10 & 10 & 10 & 20 & 80 & 80 & 160 & 190 \\
\hline Average & 10 & 10 & 20 & 68 & 79 & 146 & 192 \\
\hline \begin{tabular}{|l|} 
Average \\
Total \\
Time \\
(msec)
\end{tabular} & \multicolumn{7}{|c|}{75 (msec) } \\
\hline
\end{tabular}

In Table 5.3, Execution time for Encryption is used to calculate the throughput of Encryption scheme. It indicates the speed of encryption. The throughput of the encryption scheme is calculated by dividing the total plaintext in Megabytes encrypted by the total encryption time for each algorithm in millisec. As the throughput value is increased, the power consumption of the encryption technique is decreased [30].

- Throughput For Encryption(Megabytes/sec)=

- Throughput

- Throughput For Encryption In Proposed Algorithm = 3.9145 Megabytes/sec

- Throughput for Encryption in Proposed Algorithm is greater than compared to DES and 3DES. Hence proposed algorithm is better. 
TABLE 5.3 :Throughput Analysis between Different Algorithms for Encryption [30]

\begin{tabular}{|c|c|c|c|}
\hline Input size in (kbytes) & DES (msec) & 3DES (msec) & Proposed (msec) \\
\hline $\mathbf{4 9}$ & 29 & 54 & 10 \\
\hline $\mathbf{5 9}$ & 33 & 48 & 10 \\
\hline $\mathbf{1 0 0}$ & 49 & 81 & 20 \\
\hline $\mathbf{2 4 7}$ & 47 & 111 & 57 \\
\hline $\mathbf{3 2 1}$ & 82 & 167 & 74 \\
\hline $\mathbf{6 9 4}$ & 144 & 226 & 251 \\
\hline $\mathbf{8 9 9}$ & 240 & 299 & 84.42 \\
\hline $\begin{array}{c}\text { Ahroughput } \\
\text { Megabytes/sec) }\end{array}$ & 89.1 & 140.86 & $\mathbf{3 . 9 1 4 5}$ \\
\hline
\end{tabular}

In Fig 5.5 we have explained throughput of DES, 3DES and PROPOSED algorithms with the help of bar graph.

As a result, encryption time for proposed algorithm is better than DES and 3DES.

Less power consumption in proposed algorithm compared to DES and 3DES.

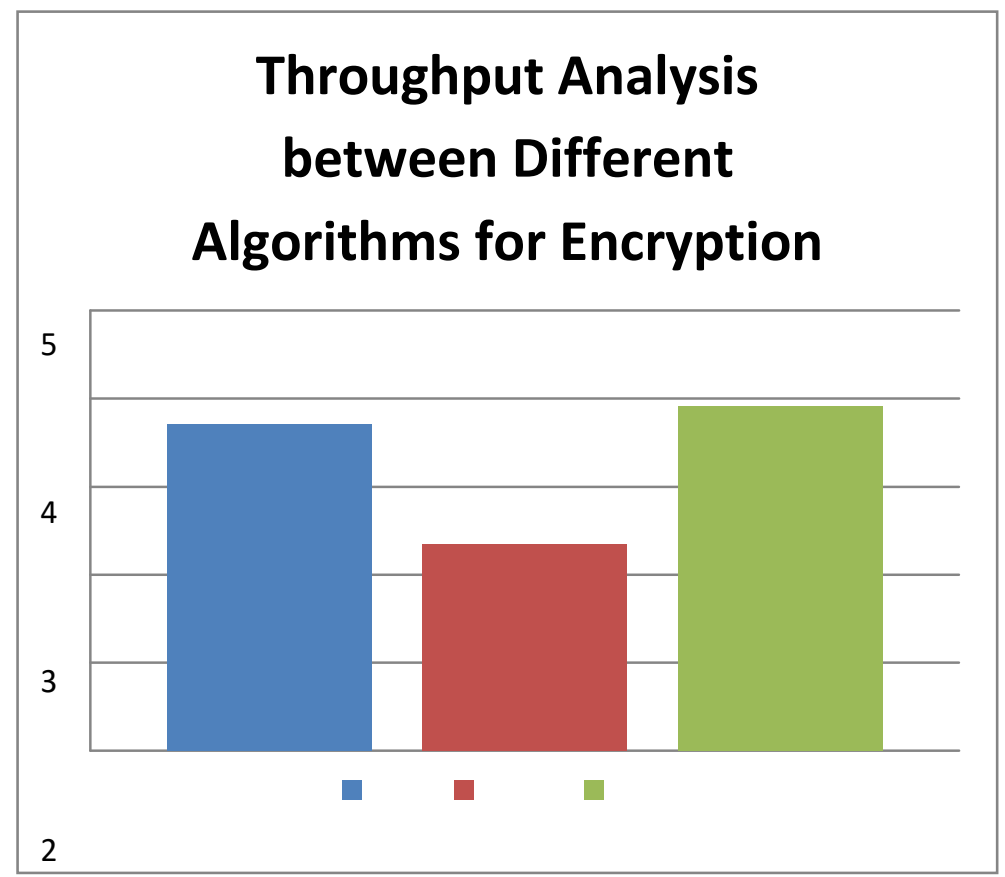

Figure 5.5 Bar Graph for Throughput in different Algorithm for Encryption

In Table 5.4, The throughput of the Decryption scheme is calculated similarly as in encryption, it indicates the speed of Decryption.

- Throughput For Decryption In Proposed Algorithm = 4.4066 Megabytes/sec 
TABLE 5.4: Throughput Analysis between Different Algorithms for Decryption [30]

\begin{tabular}{|c|c|c|c|}
\hline Input size in (kbytes) & DES (msec) & 3DES (msec) & Proposed (msec) \\
\hline $\mathbf{4 9}$ & 50 & 53 & 10 \\
\hline $\mathbf{5 9}$ & 42 & 51 & 10 \\
\hline $\mathbf{1 0 0}$ & 57 & 57 & 20 \\
\hline $\mathbf{2 4 7}$ & 72 & 77 & 68 \\
\hline $\mathbf{3 2 1}$ & 74 & 87 & 146 \\
\hline $\mathbf{6 9 4}$ & 120 & 147 & 192 \\
\hline $\mathbf{8 9 9}$ & 152 & 91.86 & 75 \\
\hline $\begin{array}{c}\text { Ahroughput } \\
\text { Megabytes/sec) }\end{array}$ & 81 & $\mathbf{3 . 5 9 7 8}$ & $\mathbf{4 . 4 0 6 6}$ \\
\hline
\end{tabular}

In Fig 5.6 we have explained throughput of DES, 3DES and PROPOSED algorithms with the help of bar graph.

As a result, decryption time for proposed algorithm is better than DES and 3DES.

Less power consumption in proposed algorithm compared to DES and 3DES.

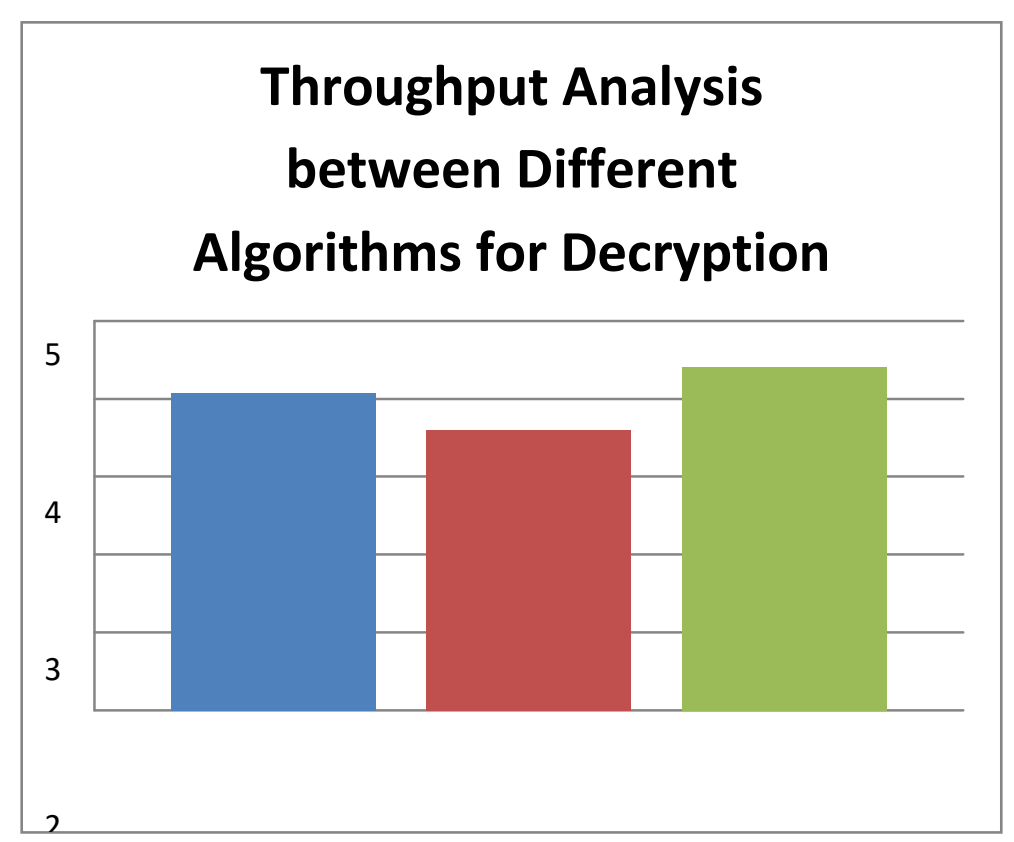

Figure 5.6 Bar Graph for Throughput in different Algorithm for Decryption

DES encrypts data in 64-bit block size and works effectively a 56 bit key. 56-bit key space amounts to approximately 72 quadrillion probabilities. It looks huge but according to today computing power it is not enough and vulnerable to brute force attack. Therefore, DES could not keep up with advancement in technology and it is no longer relevant for security. Because 
DES was extensively used at that time, the quick clarification was to combine 3DES which are secure fit for most views today. 3DES is a formation of using DES 3 times in sequence. 3DES with 3 distinct keys (K1, K2, and K3) has effective key length of 168 bits (The use of three distinct key is recommended of 3DES.). Another variation is called two-key (K1 and $\mathrm{K} 3$ are same) 3DES reduces the effective key size to 112 bits which are less secure [30].

TABLE 5.5

Comparison with existing algorithms

\begin{tabular}{|c|c|c|c|c|}
\hline S.No. & FACTORS & DES & 3DES & PROPOSED \\
\hline 1 & KEY LENGTH & 56 BITS & 168 BITS & 256 BYTE \\
\hline 2 & CIPHER TYPE & $\begin{array}{l}\text { SYMMETRIC } \\
\text { ALGORITHM }\end{array}$ & $\begin{array}{l}\text { SYMMETRIC } \\
\text { ALGORITHM }\end{array}$ & $\begin{array}{l}\text { SYMMETRIC } \\
\text { ALGORITHM }\end{array}$ \\
\hline 3 & BLOCK SIZE & 64 BITS & 64 BITS & 256 BYTE \\
\hline 4 & DEVELOPED & 1977 & 1978 & 2018 \\
\hline 5 & KEY & SINGLE & $\begin{array}{l}\text { SINGLE (Later } \\
\text { divided in } 3 \text { parts) }\end{array}$ & SINGLE \\
\hline 6 & POSSIBLE KEYS & $2^{56}$ & $2^{112}, 2^{168}$ & $2^{256 \times 8}$ \\
\hline 7 & $\begin{array}{l}\text { THROUGHPUT } \\
\text { (MB/sec) } \\
\text { (Encryption/Decryption) }\end{array}$ & $3.7074 / 4.0802$ & $2.3463 / 3.5978$ & $3.9145 / 4.4066$ \\
\hline 8 & SECURITY & $\begin{array}{l}\text { PROVEN } \\
\text { INADEQUATE }\end{array}$ & WEAK & $\begin{array}{l}\text { MORE } \\
\text { RELIABLE }\end{array}$ \\
\hline 9 & ROUND & 16 & 48 & 1 \\
\hline
\end{tabular}

As DES and 3DES proved to be insufficient in providing security and coping up with the todays computing power, our algorithm encryption and decryption appears to be more reliable, as the key length is increased, hence the possible keys increased. DES, 3DES and our proposed algorithm are symmetric algorithms which use same key for encryption and decryption. The throughput in proposed algorithm observed to be improved over existing algorithms. As throughput is inversely proportional to the power consumption, hence power consumption reduces with increasing throughput.

\subsection{Computing Power Analysis}

Total number of computations required for converting plain text to cipher text.

Computation one: Converting $n=0: 255$ to Quaternary vector. Let it be QVR.

Computation two: Calculating QVR-1 and storing it in QVR. Computation three: Multiplying QVR with the key considered. Computation four: Applying sign function on the product. Store it in QVR. Computation five: Calculating QVR+1.

Computation six: Converting output Quaternary vector to integer form. Let this be SEQ, the sequence generated.

Computation seven: Converting plain text to ASCII.

Computation eight: Adding ASCII value of plain text to sequence generated.

Computation nine: Applying mod function on the output. 
DOI: https://dx.doi.org/10.26808/rs.ca.i8v4.03 International Journal of Computer Application (2250-1797)

Issue 8 Volume 4, July-August 2018

Computation ten: Converting the output to characters of the alphabet to get cipher text. Thus the total number of computations in the first proposed model is 10 .

Computation overhead (Computing Power) for a 256 character key. $1^{\text {st }}$ computation: 256 calculations,

$2^{\text {nd }}$ computation: 256 calculations. Key considered: character key, $3^{\text {rd }}$ computation: $256 \times 16$ calculations,

$4^{\text {th }}$ computation: 256 calculations, $5^{\text {th }}$ computation: 256 calculations, $6^{\text {th }}$ computation: 256 calculations,

$7^{\text {th }}$ computation: 256 calculations. Considering a 256 character plain text, $8^{\text {th }}$ computation: 256 calculations,

$9^{\text {th }}$ computation: 256 calculations, $10^{\text {th }}$ computation: 256 calculations.

Thus the total computational overhead by this model is 6400 calculations.

\subsection{Complexity of the Model}

1. Computation: Converting n=0:256 to Quaternary vector. Let it be QVR. The complexity is in multiples of $n$.

2. Computation: Calculating QVR-1. The complexity is in multiples of $\mathrm{n}$.

3. Computation: Multiplying QVR with the key considered. Complexity is in multiples of $n$

4. Computation: Applying sign function on the product. Store it in QVR. The complexity is in multiples of $n$

5. Computation: Calculating QVR+1. The complexity is in multiples of $\mathrm{n}$

6. Computation: Converting output Quaternary vector to integer form. Let this be SEQ, the sequence generated. The complexity is in multiples of $n$

7. Computation: Converting plain text to ASCII value. The complexity is in multiples of $n$

8. Computation: Adding ASCII value of plain text to sequence generated. The complexity is in multiples of $n$

9. Computation: Applying mod function on the output. The complexity is in multiples of $n$

10. Computation: Converting the output to characters of the alphabet to get cipher text. The complexity is in multiples of $n$.

11. Thus, we can say that the complexity of model is $\mathrm{O}(\mathrm{n})$.

\section{CONCLUSION \& FUTURE WORK}

\subsection{Conclusion}

Finally, we are at the conclusion phase of this study. We have focussed in this study about cloud computing security and trust management between cloud service providers and cloud customers. This study represents the importance of Encryption and Decryption phase used in cloud security. The significance of encrypted data can be identified in light of the mushrooming applications and globalization of communication. The advantages of encrypting data in cloud computing maintains trust among the cloud customers because they want to manifest themselves for security and confidentiality in real time applications. Encryption of data is of particular significance in applications like email, e-commerce, e-cash where highly vulnerable communication lines are accessed for transmission of highly volatile data.

Encryption model presented in chapter 4 depicts new block cipher technique. The algorithm considers a matrix key and executes a sequence of steps which generates another sequence. This model is a new symmetric encryption technique. In the model, it is observed that for a given key, the total computational overhead is 1600 calculations. The complexity of the 
DOI: https://dx.doi.org/10.26808/rs.ca.i8v4.03 International Journal of Computer Application (2250-1797)

Issue 8 Volume 4, July-August 2018

model by its construction is $\mathrm{O}(\mathrm{n})$ of its strength, it is exponential in nature. Its throughput is much better compared to another algorithm hence power consumption is low. It is also observed that by slight variations in the key, a lot of variations in cipher text is identified which provides more strength to the generated algorithm. This algorithm is completely free from attacks on cipher text.

In this work, a Quaternary system with a 4 digit number is used. So the secret key generated is a $4^{4}$ i.e. a 256 digit number. By considering a Quaternary vector with a five digit number or six-digit number, the length of the secret key can be increased by $4^{5}, 4^{6}$ which increase the length of secret key generated. Similarly by considering $\mathrm{n}$-ary vector the length of the secret key generated can still be increased. Thus by increasing the length of secret-key, security of cipher system can be increased still further.

\subsection{Future Work}

The present work deals with plain text being represented in numerical and characters of English alphabet. This work can be improved so that it can support the characters of not only English but also of other languages as well. This work can also be improved to support not only text but also other forms of message transmission like audio, video and images. We will analyze performance of other algorithms with respect to throughput and power consumption.

\section{REFERENCES}

I. R. Buyya, C. Shin Yeo, J. Broberg, and I. Brandic, "Cloud computing and emerging it platforms: Vision, hype, and reality for delivering computing as the 5th utility," Future Generation Computing System, vol. 25, pp. 599-616, 2009.

II. Web Reference from http://csrc.nist.gov/publications/nistpubs/800-145/SP800-145.pdf.

III. Ronald L. Krutz, Russell Dean Vines,"Cloud Security: A Comprehensive Guide to Secure Cloud Computing", ISBN: 978-0-470-58987-8, Wiley Publishing, Inc.

IV. William Stallings, "Cryptography and Network Security Principles and Practice", ISBN 978-9332518773, Pearson Education India, 6th edition.

V. Khaled M Khan and Qutaibah Malluhi, "Establishing Trust in Cloud Computing," IT Professional, vol. 12, no. 5, pp. 20 - 27, 2010.

VI. Zhexuan Song, Jusus Molina, and Christina Strong, "Trusted Anonymous Execution: A Model to Raise Trust in Cloud," in 9th International Conference on Grid and Cooperative Computing (GCC), Nanjing, China, 2010, pp. 133 - 138.

VII. Hiroyuki Sato, Atsushi Kanai, and Shigeaki Tanimoto, "A Cloud Trust Model in a Security Aware Cloud," in 10th IEEE/IPSJ International Symposium on Applications and the Internet (SAINT), Seoul, South Korea, 2010, pp. 121 - 124.

VIII. Wenjuan Li, Lingdi Ping, and Xuezeng Pan, "Use trust management module to achieve effective security mechanisms in cloud environment," in International Conference on Electronics and Information Engineering (ICEIE), vol. 1, Kyoto, Japan, 2010, pp. 14- 19.

IX. Tie Fang Wang, Bao Sheng Ye, Yun Wen Li, and Yi Yang, "Family Gene based Cloud Trust Model," in International Conference on Educational and Network Technology (ICENT), Qinhuangdao, China, 2010, pp. 540 - 544.

X. Tie Fang Wang, Bao Sheng Ye, Yun Wen Li, and Li Shang Zhu, "Study on Enhancing Performance of Cloud Trust Model with Family Gene Technology," in 3rd IEEE International Conference on Computer Science and Information Technology (ICCSIT), vol. 9, Chengdu, China, 2010, pp. $122-126$.

XI. Paul D Manuel, Thamarai Selve, and Mostafa Ibrahim Abd-EI Barr, "Trust management system for grid and cloud resources," in First International Conference on Advanced 
DOI: https://dx.doi.org/10.26808/rs.ca.i8v4.03 International Journal of Computer Application (2250-1797)

Issue 8 Volume 4, July-August 2018

Computing (ICAC 2009), Chennai, India,2009, pp. 176-181.

XII. Zhidong Shen, Li Li, Fei Yan, and Xiaoping Wu, "Cloud Computing System Based on Trusted Computing Platform," in International Conference on Intelligent Computation Technology and Automation (ICICTA), vol. 1,Changsha, China, 2010, pp. 942 - 945.

XIII. Zhidong Shen and Qiang Tong, "The security of cloud computing system enabled by trusted computing technology," in 2nd International Conference on Signal Processing Systems (ICSPS), vol. 2, Dalian, China, 2010, pp. 11-15.

XIV. Mohammed Alhamad, Tharam Dillon, and Elizabeth Chang, "SLA-based Trust Model for Cloud Computing," in 13th International Conference on Network-Based Information Systems, Takayama, Japan, 2010, pp. 321 -324.

XV. Xiao Yong Li, Li Tao Zhou, Yong Shi, and Yu Guo, "A trusted computing environment model in cloud architecture," in Ninth International Conference on Machine Learning and Cybernetics (ICMLC), vol. 6, Qingdao, China,2010, pp. 2843-2848.

XVI. Zhimin Yang, Lixiang Qiao, Chang Liu, Chi Yang, and Guangming Wan, "A CollFaborative Trust Model of Firewall-through based on Cloud Computing," in 14th International Conference on Computer Supported Cooperative Work in Design (CSCWD), Shanghai, China, 2010, pp. 329 - 334.

XVII. Junning Fu, Chaokun Wang, Zhiwei Yu, Jianmin Wang, and Jia Guang Sun, "A Watermark-Aware Trusted Running Environment for Software Clouds," in Fifth Annual China Grid Conference (China Grid), Guangzhou, China,2010, pp. 144 - 151.

XVIII. Rohit Ranchal et al., "Protection of Identity Information in Cloud Computing without Trusted Third Party," in 29th IEEE International Symposium on Reliable Distributed Systems, New Delhi, India, 2010, pp. 1060-9857.

XIX. Hassan Takabi, James B.D Joshi, and Gail Joon Ahn, "Secure Cloud: Towards a Comprehensive Security Framework for Cloud Computing Environments," in 34th Annual IEEE Computer Software and Applications Conference Workshops, Seoul, South Korea, 2010, pp. 393 - 398.

XX. Web reference from https://en.wikipedia.org/wiki/DataEncryptionStandard.

XXI. Atul Kahate, "Cryptography and Network Security", ISBN 1-25-902988-3, McGraw Hill Education (India) Private Limited, $3^{\text {rd }}$ edition.

XXII. Sombir Singh, Sunil K.Makkar and Dr.Mukesh Kumar, "Enhancing the Security of DES Algorithm Using Transposition Cryptography Techniques" in IJARCSSE in volume 3, issue 6,BRCM CET, Bahal, India 2013, ISSN: 2277 128X.

XXIII. Des encryption by National Institute of Standards and Technology (NIST) chapter 6.

XXIV. Li, J., Li, B., Meng, L., \& Sun, D, "HiTrust: A hybrid tree based trust negotiation service" IEEE 24th International Conference on Advanced Information Networking and Application Workshops, 2010. doi:10.1109/WAINA.2010.149.

XXV. Kramer, S., Gore, R., \& Okamoto, E, "Formal dentitions and complexity results for trust relations and trust domains" March 2012, from http://www1.spms.ntu.edu.sg/ ccrg/documents/trust.pdf.

XXVI. Abawajy, J,'Determining service trustworthiness in inter cloud computing environments". 10th International Symposium on Pervasive Systems, Algorithms,and Networks,2009, doi:10.1109/I-SPAN.2009.155.

XXVII. Habib, M. H., Reis, S., \& Muhlhauser, M, “Towards a trust management system for cloud computing" International joint conference of IEEETrustCom-11/IEEE ICESS11/FCST-11, 2011, doi:10.1109/TrustCon.2011.129.

XXVIII.Skogsrud, H. \& Benatallah, B, "Model-driven trust negotiation for web services. 
IEEE Internet Computing”.2003, doi:10.1109/MIC.2003.1250583.

XXIX. Kerr, R. \& Cohen, R, "Smart cheaters do prosper: Defeating trust and reputation systems.", 8th International Conference on Autonomous Agents and Multiagent Systems,March 2012, from www.cs.uwaterloo.ca/rckerr/KerrCohenaamas2009draft.pdf.

XXX. Milind Mathur and Ayush, "Comparison Between DES , 3DES , RC2 , RC6 , BLOWFISH AND AES “, National Conference on New Horizons in IT,2013.

XXXI. G. Ramesh and R. Umarani," A Comparative Study of Six Most Common Symmetric Encryption Algorithms across Different Platforms “, International journal of Computer Application, ISSN: 0975 - 8887, Volume 46- No.13, May 2012.

XXXII. Saket Maskara1, Mudit Saraf and Priya G," Trust Management in Cloud Computing", International Research Journal of Engineering and Technology, ISSN: 2395-0072, Volume: 03 Issue: 11, Nov -2016.

XXXIII. R.Gowthami Saranya and A.Kousalya," A Comparative Analysis of Security Algorithms Using Cryptographic Techniques in Cloud Computing", International Journal of Computer Science and Information Technologies, ISSN: 0975-9646, Vol. 8 (2), 2017, 306-310. 